\title{
Factores asociados a la integración laboral de las personas en tratamiento renal sustitutivo en España
}

\author{
Juan Carlos Julián-Mauro, Sara Muñoz-Carrasquilla, Inés Rosado-Lázaro \\ Fundación Renal ALCER. Madrid. España
}

\begin{abstract}
Como citar este artículo:
Julián-Mauro JC, Muñoz-Carrasquilla S, Rosado-Lázaro I.

Factores asociados a la integración laboral de las personas en tratamiento renal sustitutivo en España.

Enferm Nefrol. 2020 Abr-Jun;23(2).176-83
\end{abstract}

\section{Resumen}

Introducción: Las personas en tratamiento renal sustitutivo en edad laboral presentan tradicionalmente tasas de empleo significativamente más bajas que la población general.

Objetivo: Describir la situación laboral de las personas con enfermedad renal crónica en tratamiento renal sustitutivo en España e identificar los factores asociados más significativos.

Material y Método: Estudio observacional descriptivo trasversal comparativo de personas en los diferentes tratamientos renales sustitutivos, en edad laboral de 16 a 64 años. Se analizó su situación laboral y otros factores socio-demográficos y socio-económicos, mediante la administración de un cuestionario ad hoc.

Resultados: En 437 personas con enfermedad renal crónica avanzada en tratamiento con hemodiálisis, diálisis peritoneal o trasplante. La tasa de empleo fue del $27 \%$, un $84,21 \%$ de personas tenían certificado de discapacidad y un $46,45 \%$ de incapacidad laboral reconocida. Las personas trasplantadas presentaron mayores ingresos económicos mensuales y las que estaban en diálisis domiciliarios tenían la mayor tasa de empleo $(37,64 \%$ frente al $27,89 \%$ de las personas trasplantadas y el $20,37 \%$ de las personas en hemodiálisis en centro).

Conclusiones: Las personas en tratamiento renal sustitutivo en edad laboral tienen importantes limitaciones

\section{Correspondencia:}

Juan Carlos Julián Mauro

Email: jcjulian@alcer.org en su acceso al empleo, sin embargo, existen diferencias importantes según sea la opción elegida de tratamiento. La información y toma de decisiones respecto opción del tratamiento renal sustitutivo son claves para el mantenimiento u obtención de un empleo, para su situación económica futura e incluso para el reconocimiento de la incapacidad laboral.

PALABRAS CLAVE: hemodiálisis; diálisis peritoneal; trasplante renal; empleo; discapacidad; incapacidad laboral.

\section{Factors associated with the labor integration of people on renal replacement therapy in Spain}

\section{Abstract}

Introduction: People on renal replacement therapy at working age traditionally have significantly lower employment rates than the general population.

Objective: To describe the employment situation of people with chronic kidney disease on renal replacement therapy in Spain and to identify the significant associated factors.

Material and Method: Observational, descriptive, cross-sectional and comparative study of people in the different renal replacement treatments, during the work period of 16 to 64 years. Their employment situation and other socio-demographic and socioeconomic factors were analysed through the use of an ad hoc questionnaire.

Results: In 437 people with advanced chronic kidney disease on haemodialysis, peritoneal dialysis 
or transplantation. The employment rate was $27 \%$, $84.21 \%$ of people had a certificate of disability and $46.45 \%$ had a recognized incapacity for work. The transplanted persons presented higher monthly economic income. Patients receiving home dialysis had a higher employment rate $37.64 \%$ compared to $27.89 \%$ of transplant recipients, and $20.37 \%$ of people on centre haemodialysis).

Conclusions: People in renal replacement therapy at working age have significant limitations in access to employment. Although, there are important differences depending on the treatment option chosen. Information and decision-making regarding the option of renal replacement treatment are key for maintaining or obtaining a job, for the future economic situation, and even for the recognition of incapacity for work.

KEYWORDS: haemodialysis; peritoneal dialysis; kidney transplant; employment; disability; incapacity for work.

\section{Introducción}

Las personas en tratamiento renal sustitutivo (TRS) en edad laboral presentan tradicionalmente tasas de empleo significativamente más bajas que la población general ${ }^{1,2}$, muy próximas al resto de personas con discapacidad ${ }^{3-6}$. La integración laboral de las personas con discapacidad es uno de los retos que nuestra sociedad lleva afrontando desde hace décadas y dónde las personas en TRS tienen especiales dificultades.

La población española presenta una de las tasas de envejecimiento más altas del mundo, con una población en edad laboral muy reducida ${ }^{2}$ y dónde se plantea como reto incorporar a las personas con enfermedad y patologías crónicas en edad laboral al mercado de trabajo. Las nuevas tecnologías y procesos industriales hacen cada vez más viables las posibilidades de que estas personas se incorporen al proceso productivo. Existen programas financiados por la unión Europea que tratan de informar a los empleadores sobre la capacidad de los pacientes crónicos para poder ser contratados y trabajar ${ }^{7}$. En contra de esta integración juegan las altas tasas de desempleo que arrastramos en España.

Los tratamientos renales sustitutivos han permitido alargar la supervivencia de las personas de forma considerable, pero suponen un alto coste para el sistema público de salud $^{8}$ (alrededor de un 2,5\% del coste total para sólo un $0,1 \%$ de la población). A ello se añaden los costes socia- les añadidos ${ }^{9}$ que están relacionados con la condición de pensionista, en personas en edad laboral. Por todo ello resulta necesario conocer cuáles son los factores que influyen en el acceso al empleo de estas personas, sus tasas de discapacidad e incapacidad laboral y qué influencia tienen los diferentes tratamientos sustitutivos en las tasas de empleo y en sus ingresos económicos.

En estudios previos se han identificado las técnicas domiciliarias ${ }^{10,11}$ y el tratamiento de la anemia prediálisis ${ }^{12}$ como factores clave en el mantenimiento del empleo en las personas que comienzan TRS. En anteriores estudios de nuestro grupo son las personas en tratamiento domiciliario con diálisis peritoneal las que presentaban mayor tasa de empleo ${ }^{1}$.

Los profesionales enfermeros son tradicionalmente los encargados de realizar los procesos informativos y educativos sobre las opciones de TRS y también los que más próximos están a los pacientes ${ }^{13}$. Habitualmente ofrecen también apoyo e información en ámbitos socio-sanitarios ${ }^{14}$, que pueden influir en la toma de decisiones del paciente respecto a su futura actividad.

Por todo ello, consideramos que es importante conocer qué factores influyen en la integración laboral de las personas en TRS y qué servicios existen para ayudarles. Esta información puede ayudarles a mantener o encontrar un empleo y mejorar su situación socioeconómica y de calidad de vida.

Los objetivos de nuestra investigación fueron describir la situación laboral de las personas con enfermedad renal crónica en tratamiento renal sustitutivo en España e identificar los factores más significativos que determinan su integración socio-laboral.

\section{Material y Método}

Estudio observacional descriptivo trasversal comparativo de personas en las siguientes modalidades de TRS: hemodiálisis en centros sanitarios, diálisis domiciliaria (diálisis peritoneal o hemodiálisis domiciliaria) o trasplante renal.

El ámbito de estudio fue estatal, desde enero de 2018 hasta marzo de 2019, colaborando las Asociaciones para la Lucha Contra las Enfermedades Renales (ALCER) de las provincias de Alicante, Badajoz, Barcelona, Cáceres, Ciudad Real, Coruña, Lugo, Pontevedra, Ourense, Sevilla, Granada, Illes Balears, Jaén, Madrid, Málaga, Huelva y Toledo. 
Como criterio de inclusión para participar en el estudio se definió las personas con enfermedad renal crónica avanzada, en TRS y en edad laboral (entre 16 y 64 años). Se descartaron aquellos casos que, hubieran comenzado un proceso de pre-jubilación laboral.

Para la realización de este estudio se diseñó un cuestionario ad-hoc, validados por expertos, donde se ha recogido información sobre las siguientes variables:

- Demográficas (edad, sexo).

- Socio-económicas (actividad, ingresos económicos, prestaciones sociales).

- Laborales (en activo, incapacidad laboral).

- Sanitarias: (tipo de TRS, grado discapacidad).

La Federación Nacional de Asociaciones ALCER distribuyó los cuestionarios entre las personas participantes que desearon participar, cumplimentándolos solos o con la ayuda del trabajador/a social de la entidad.

Para la inclusión en el estudio se recabó consentimiento verbal, después de la lectura del texto que aparece al inicio del cuestionario y entendiendo que la auto cumplimentación del mismo implicaba la aceptación de participar en el estudio. No se han incluido datos de carácter personal que pudieran identificar a los pacientes, respetando los principios éticos y universales, así como las normas internacionales de protección de datos y la legislación española vigente.

El análisis estadístico se hizo mediante el paquete SPSS v.24 para generar una de tablas y correlaciones que permitió comparar la situación de la persona con ERC con otros grupos y subgrupos sociales, atendiendo a distintas categorías de análisis. Se han utilizado pruebas $t$ de Student de comparación de medias de dos muestras independientes para el estudio de la relación con variables continuas. Se ha considerado un $p$ valor $<0,05$ como estadísticamente significativo.

\section{Resultados}

Respondieron al cuestionario 437 sujetos en edad laboral, socia/os y usuaria/os de los servicios provistos por ALCER. En la Tabla 1 se puede consultar las características principales de los sujetos participantes.

Tabla 1. Características socio-laborales de la muestra.

\begin{tabular}{|c|c|c|c|c|c|}
\hline & & Hombres (n) & Mujeres (n) & Total (n) & Tasa (\%) \\
\hline Edad & $\begin{array}{l}16-35 \text { años } \\
36-55 \text { años } \\
56-64 \text { años }\end{array}$ & $\begin{array}{c}29 \\
151 \\
80\end{array}$ & $\begin{array}{c}15 \\
111 \\
51\end{array}$ & $\begin{array}{c}44 \\
262 \\
131\end{array}$ & $\begin{array}{l}10,07 \% \\
59,95 \% \\
29,98 \%\end{array}$ \\
\hline Formación & $\begin{array}{l}\text { Sin Estudios } \\
\text { Primaria } \\
\text { Secundaria } \\
\text { Universitaria } \\
\text { NS/NC }\end{array}$ & $\begin{array}{c}5 \\
99 \\
96 \\
59 \\
1\end{array}$ & $\begin{array}{c}11 \\
70 \\
56 \\
40 \\
0\end{array}$ & $\begin{array}{c}16 \\
169 \\
152 \\
99 \\
1\end{array}$ & $\begin{array}{c}3,66 \% \\
38,67 \% \\
34,78 \% \\
22,65 \% \\
0,23 \%\end{array}$ \\
\hline TRS & $\begin{array}{l}\text { HD en Centro } \\
\text { Diálisis Domiciliaria } \\
\text { Trasplante renal }\end{array}$ & $\begin{array}{c}103 \\
48 \\
109\end{array}$ & $\begin{array}{l}59 \\
37 \\
81\end{array}$ & $\begin{array}{c}162 \\
85 \\
190\end{array}$ & $\begin{array}{l}37,07 \% \\
19,45 \% \\
43,48 \%\end{array}$ \\
\hline Discapacidad & $\begin{array}{l}\text { Sin Certificado } \\
33 \%-64 \% \\
65 \%-74 \% \\
75 \% \text { ó más }\end{array}$ & $\begin{array}{l}48 \\
83 \\
97 \\
32\end{array}$ & $\begin{array}{l}21 \\
49 \\
81 \\
26\end{array}$ & $\begin{array}{c}69 \\
132 \\
178 \\
58\end{array}$ & $\begin{array}{l}15,79 \% \\
30,21 \% \\
40,73 \% \\
13,27 \%\end{array}$ \\
\hline Ingresos netos mensuales & $\begin{array}{l}\text { Sin Ingresos } \\
\text { Menos } 300 € \\
\text { De } 301 € \text { a } 500 € \\
\text { De } 501 € \text { a } 1000 € \\
\text { De } 1001 € \text { a } 1500 € \\
\text { De 1501€ a } 2000 € \\
\text { Más de } 2001 € \\
\text { NS/NC }\end{array}$ & $\begin{array}{c}7 \\
3 \\
27 \\
87 \\
78 \\
29 \\
23 \\
6\end{array}$ & $\begin{array}{c}23 \\
6 \\
38 \\
67 \\
29 \\
12 \\
1 \\
1\end{array}$ & $\begin{array}{c}30 \\
9 \\
65 \\
154 \\
107 \\
41 \\
24 \\
7\end{array}$ & $\begin{array}{c}6,86 \% \\
2,06 \% \\
14,87 \% \\
35,24 \% \\
24,49 \% \\
9,38 \% \\
5,49 \% \\
1,60 \%\end{array}$ \\
\hline Situación Socio-laboral & $\begin{array}{l}\text { PC } \\
\text { PNC } \\
\text { Trabajando } \\
\text { Desempelado/a } \\
\text { NS/NC }\end{array}$ & $\begin{array}{c}133 \\
13 \\
74 \\
40 \\
0\end{array}$ & $\begin{array}{c}70 \\
20 \\
44 \\
39 \\
4\end{array}$ & $\begin{array}{c}203 \\
33 \\
118 \\
79 \\
4\end{array}$ & $\begin{array}{c}46,45 \% \\
7,55 \% \\
27,00 \% \\
18,08 \% \\
0,92 \%\end{array}$ \\
\hline
\end{tabular}


El $51,25 \%$ de los participantes habían obtenido la calificación de incapacidad laboral tras haber iniciado TRS, en la Figura 1, puede observarse la distribución de los tipos de incapacidad, destacando la incapacidad permanente absoluta $(69,73 \%)$.

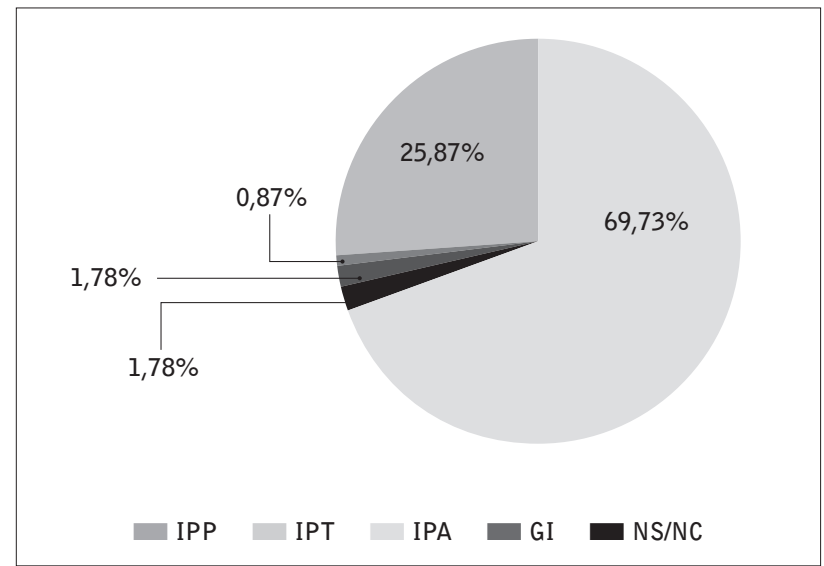

Figura 1. Distribución según tipo de incapacidad laboral.

IPP: incapacidad permanente parcial; IPT: incapacidad permanente total; IPA: incapacidad permanente absoluta; GI: gran incapacidad; NS/NC: no sabe/no contesta.

El $84,21 \%$ poseía certificado de discapacidad igual o superior al 33\% (mínimo legal para ser considerada persona con discapacidad) y atendiendo al grado de discapacidad el $36,86 \%$ tenían un grado de discapacidad entre el 33 y $64 \%$, el $48,36 \%$ entre $65-74 \%$ y el 15,76\% más del 75\% (Tabla 2).

Dentro de los subgrupos de incapacidad la situación laboral es la siguiente:

- Con incapacidad permanente parcial (IPP) trabajaba el $100 \%$.
- Con incapacidad permanente total (IPT) trabajaba el $13,55 \%$.

- Con incapacidad permanente absoluta (IPA) trabajaba el 0,62\% mientras que el 99,38\% no lo hacía.

- Con gran incapacidad (GI) no trabajaba el $100 \%$.

Los resultados en función de su situación laboral y su certificado de discapacidad, están representados en la Figura 2, las personas con certificado de discapacidad, mayoritariamente perciben una pensión. La población activa laboralmente tiene mayor número de personas sin discapacidad, en comparación con las personas que cobran pensiones. La población activa laboralmente, tienen mayor número de personas sin discapacidad, en comparación con las personas que cobran pensiones.

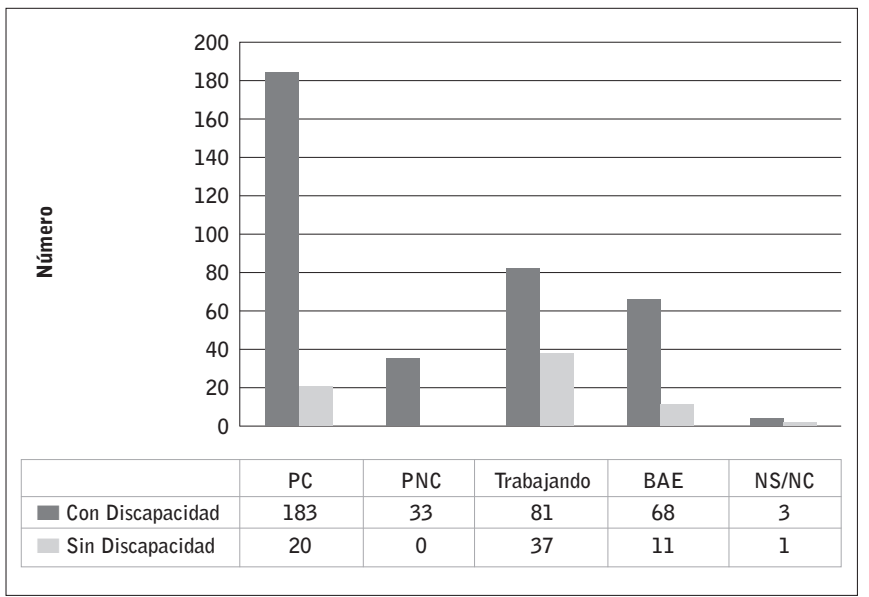

Figura 2. Discapacidad reconocida según Situación Laboral.

PC: pensión contributiva, PNC: pensión no contributiva, BAE: búsqueda activa de empleo y NS/NC no sabe, no contesta.

Tabla 2. Incapacidad laboral y grado de discapacidad según tipo de tratamiento renal sustitutivo.

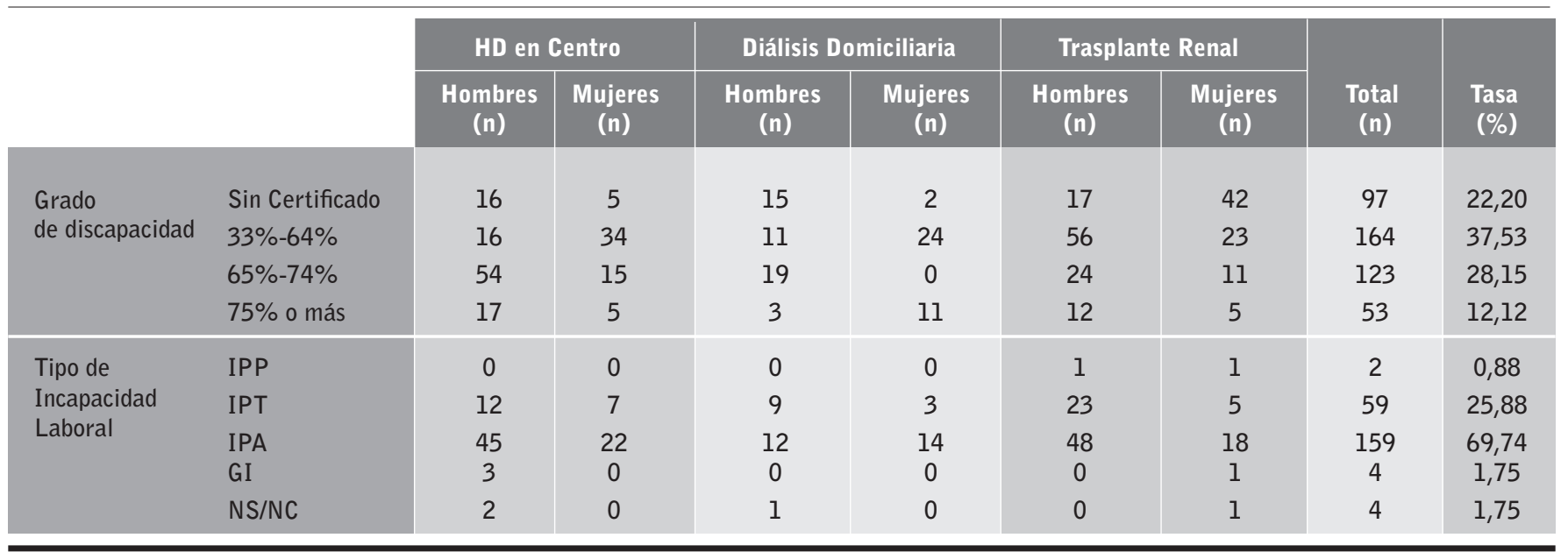

IPP: incapacidad permanente parcial; IPT: incapacidad permanente total; IPA: incapacidad permanente absoluta; GI: gran incapacidad; NS/NC: no sabe/no contesta. 
Comparando los ingresos mensuales con la situación del paciente en relación al tipo de TRS (Tabla 3), los sujetos trasplantados son los que más ingresos medios perciben (el 53,2\%, más de $1.000 €$ mensuales), seguidos por los que están en tratamiento de hemodiálisis en centro sanitario $(45,1 \%)$ y, por último, aquellos en tratamiento de diálisis domiciliario (41,2\% más de $1.000 €$ mensuales). Existen diferencias estadísticamente significativas $(p \leq 0,05)$ entre los ingresos de las personas en trasplante renal y las que se encuentran en tratamientos de diálisis, no encontrándose diferencias estadísticamente significativas en los ingresos de las personas en tratamiento de HD en centro sanitario versus diálisis domiciliaria.

Al estudiar la relación entre discapacidad reconocida e ingresos netos mensuales, parece apreciarse una tendencia de a mayor ingresos mayor porcentaje de personas con discapacidad reconocida.

En cuanto a la situación laboral de las personas en TRS, son las personas en diálisis domiciliaria las que mayor tasa de empleo tienen $(37,64 \%)$, seguidas de las que está con trasplante renal funcionante $(27,89 \%)$ y por último las que se encuentran en hemodiálisis en centro $(20,37 \%)$. Encontramos que las diferencias son estadísticamente significativas $(p \leq 0,05)$ entre las tasas de empleo de las personas en diálisis domiciliaría y trasplante renal frente a las personas en HD en centro, no habiendo significación entre las tasas de empleo de personas en diálisis domiciliaria y personas trasplantadas renales. Por último, señalar que el $74,75 \%$ de nuestra muestra indica haber dejado su puesto de trabajo anterior por motivos directamente relacionados con la ERC.

\section{Discusión}

Si comparamos nuestros resultados con los del Informe del Mercado de Trabajo de las Personas con Discapacidad ${ }^{6}$, observamos cómo la población activa de personas con discapacidad, independientemente de si tienen ERC o no, es prácticamente la misma, aunque la tasa de paro de los sujetos con discapacidad y ERC es diez puntos porcentuales más alta que la de los sujetos con discapacidad y sin ERC.

Si comparamos algunas de las cifras presentadas con el Informe Olivenza ${ }^{3}$ observamos como nuestros datos son similares a la situación general de las personas con discapacidad en España. Por una parte, encontramos más hombres con discapacidad y ERC que mujeres. Se trata de una población con una edad media de 49 años, siendo la población más joven la que más trabaja, pero por una diferencia poco significativa.

También encontramos ciertas particularidades que diferencian a nuestro grupo de grandes estudios relativos a la situación de las personas con discapacidad ${ }^{3,5}$, a) en nuestra muestra la media del grado de discapacidad se sitúa en los grados comprendidos entre el $65-75 \%$, y más alta que en otros estudios, b) la tasa de paro es más elevado, tanto en el caso de pacientes con ERC con certificado de discapacidad como sin él y c) las cifras estimadas de salario anual bruto de nuestra población, tanto con o sin certificado de discapacidad, se sitúan por debajo de la media recogida por los citados estudios 3,5 .

Son muchas las barreras a las que se enfrentan las personas en TRS en edad laboral para mantener o

Tabla 3. Ingresos económicos según tratamiento renal sustitutivo.

\begin{tabular}{|c|c|c|c|c|c|}
\hline & & \multicolumn{3}{|c|}{ TIPO TRATAMIENTO RENAL SUSTITUTIVO } & \multirow[b]{2}{*}{ Total $(n)$} \\
\hline & & HD en centro $(n)$ & Diálisis Domiciliaria ( $\mathrm{n}$ ) & Trasplante Renal (n) & \\
\hline \multirow[t]{8}{*}{ INGRESOS } & $<300 €$ & 5 & 2 & 2 & 9 \\
\hline & $301-500 €$ & 30 & 13 & 22 & 65 \\
\hline & $501-1000 €$ & 54 & 35 & 65 & 154 \\
\hline & $1001-1500 €$ & 36 & 18 & 53 & 107 \\
\hline & $1501-2000 €$ & 14 & 6 & 21 & 41 \\
\hline & $>2001 €$ & 12 & 2 & 10 & 24 \\
\hline & No tiene ingresos & 8 & 7 & 15 & 30 \\
\hline & NC & 3 & 2 & 2 & 7 \\
\hline Total & & 162 & 85 & 190 & 437 \\
\hline
\end{tabular}


conseguir un empleo y más de la mitad de la muestra $(46,45 \%)$ tiene alguna incapacidad que le impide acceder a un puesto de trabajo. Se trata de un colectivo que recibe mayoritariamente pensiones y ayudas sociales y con niveles de ingresos bajos. En esta situación destacan las personas en HD en centro son la población con mayores porcentajes de discapacidad, con más incapacidad laboral, con menos ingresos y menos población activa y empleada tienen.

Sabemos que las personas en TRS de diálisis tienen altas tasas de problemas emocionales y que ello afecta a su calidad de vida y que uno de los factores que influyen en ésta última es precisamente la actividad laboral15-17.

Es probable que el mal estado emocional (ansiedad/ depresión/estrés) esté detrás de la baja tasa de empleo de las personas en tratamiento de HD en centro y que ésta también sea causa de su peor calidad de vida. Se ha evidenciado que uno de los problemas es que las personas están desempleadas antes de entrar en diálisis y que, por tanto, un factor clave es no perder el empleo antes de iniciar diálisis ${ }^{18}$.

No todo es negativo y, por ejemplo, el análisis de series históricas en EE UU, muestran que las tasas de empleo de las personas en tratamiento de diálisis van creciendo década a década ${ }^{19}$, probablemente influido por la mayor calidad de vida que ofrecen las técnicas de diálisis, las cada vez mejores opciones de diálisis domiciliarias y la mayor atención a los problemas emocionales en las personas en TRS. Se ha relacionado también el mantenimiento del empleo al inicio de la diálisis, con disponer de opción de HD por la tarde, después de las 17 horas y con el acceso a opciones domiciliarias ${ }^{19,20}$, este hecho es compatible con nuestros datos, dónde las opciones domiciliarias alcanzan las mayores tasas de empleo $(34,9 \%)$ significativamente mayores que en HD en centro y que trasplante renal.

Sí parece claro que la propia técnica de HD en centro sanitario es una barrera para la consecución de un empleo, siempre con tasas menores que el resto de opciones de tratamiento como muestran de manera significativa varios estudios ${ }^{1,18,20,21}$, pero ofrecer horarios flexibles (y más atención a las necesidades laborales y emocionales, ansiedad/depresión, pueden ser de gran ayuda ${ }^{22,23}$. En este sentido, el creciente análisis por parte de los profesionales de la enfermería de estos aspectos emocionales y su interferencia en la calidad de vida de las personas en TRS 24,25 , muestra el interés en la necesidad de integración laboral.
Aunque la población activa es prácticamente la misma en el tratamiento de diálisis domiciliaria y de trasplante renal, las personas trasplantadas tienen mayor dificultad en encontrar empleo y por ello presentan una tasa de paro mayor y son los que engloban a más sujetos en búsqueda activa de empleo.

Este estudio presenta algunas limitaciones que debemos informar: el estudio se realiza sobre las personas que se acercan a las asociaciones ALCER, con independencia si son socios o no, pero que puede suponer un sesgo de selección. Por otro lado, analizamos la situación laboral en función del TRS, pero sin explorar cómo ha sido la situación previa, lo que puede repercutir en la interpretación de las conclusiones, ya que el estado inicial de empleabilidad puede influir en el mantenimiento del empleo con independencia de la opción de TRS de la que se trate, especialmente en la transición de diálisis a trasplante renal.

Como conclusión podemos afirmar que las personas en TRS en edad laboral tienen importantes limitaciones en su acceso al empleo, sin embargo, existen diferencias importantes según sea la opción de tratamiento elegida. Por ello los procesos de información y toma de decisiones al inicio del TRS podrían ser aspectos claves para el mantenimiento u obtención de un empleo, para su situación económica futura e incluso para el reconocimiento de su incapacidad laboral.

\section{Agradecimientos}

A las/los trabajadoras/res sociales de las entidades ALCER de Alicante: Badajoz, Barcelona, Cáceres, Ciudad Real, Coruna (A), Lugo, Pontevedra, Ourense, Sevilla (Giralda), Granada, Illes Balears, Jaén, Madrid, Málaga, Huelva (Onuba) y Toledo.

Recibido: 21-03-20

Revisado: 25-03-20

Modificado: 10-04-20

Aceptado: 25-04-20 


\section{Bibliografía}

1. Julián Mauro JC, Molinuevo Tobalina JA, Sánchez González JC. La situación laboral del paciente con enfermedad renal crónica en función del tratamiento sustitutivo renal. Nefrología. 2012;32(4):439-45.

2. Instituto Nacional de Estadística (INE). El Empleo de las Personas con Discapacidad. Año 2018. [Consultado 04 mar 2020]. Disponible en: https://www. ine.es/prensa/epd_2018.pdf.

3. Jiménez Lara A, Huete García A. Informe Olivenza 2017, sobre la situación general de la discapacidad en España. Observatorio Estatal de la Discapacidad. 2017. [Consultado 02 feb 2020]. Disponible en: www.observatoriodeladiscapacidad.info.

4. Flores N, Jenaro C, González-Gil F, García Calvo PM. Análisis de la calidad de vida laboral en trabajadores con discapacidad. EKAINA 2010;47:95107.

5. ODISMET. (2016). Módulo 1. Integración laboral y tendencias del mercado de trabajo. 2019, de ONCE. [Consultado 02 feb 2020]. Disponible en: https:// www.odismet.es/banco-de-datos/lintegracion-laboral-y-tendencias-del-mercado-de-trabajo.

6. Servicio Público de Empleo Estatal (SEPE). Informe del Mercado de Trabajo de las Personas con Discapacidad Estatal. Madrid. Observatorio de Ocupaciones 2019. [Consultado 05 feb 2020]. Disponible en: http://www.sepe.es/contenidos/observatorio/ mercado_trabajo/3206-1.pdf.

7. Chrodis + Program. Work package 8: Chronic Disease and Employment. [Consultado 05 feb 2020]. Disponible en: http://chrodis.eu/08-employment/.

8. Ministerio de Sanidad, Consumo y Bienestar Social. Documento Marco sobre Enfermedad Renal Crónica (ERC) dentro de la Estrategia de Abordaje a la Cronicidad en el SNS. 2015. [Consultado 02 feb 2020]. Disponible en: https://www.mscbs.gob. es/organizacion/sns/planCalidadSNS/pdf/Enfermedad_Renal_Cronica_2015.pdf.

9. Julián-Mauro JC, Cuervo J, Rebollo P, Callejo D. Situación laboral y costes indirectos en pacientes con insuficiencia renal: diferencias entre distintas modalidades de tratamiento renal sustitutivo. Nefrología 2013;33(3):333-41.

10. Pastor JL, Julián-Mauro JC. Claves del proceso de información y elección de modalidad de diálisis en pacientes con insuficiencia renal crónica. Nefrología 2010;1(Supl Ext 1):15-20.

11. Selgas R, Rodríguez L, Julián-Mauro JC, Remon C, Prieto-Velasco $\mathrm{M}$, Pérez-Contreras $\mathrm{J}$ et al. How a Bottom-Up Multi-Stakeholder Initiative Helped Transform the Renal Replacement Therapy Landscape in Spain. Appl Health Econ Health Policy. 2017;15(6):755-62.

12. Muehrer RJ, Schatell D, Witten D, Gangnon R, Becker RN and Hofmann RM. Factors Affecting Employment at Initiation of Dialysis. Clin $\mathrm{J} \mathrm{Am} \mathrm{Soc}$ Nephrol. 2011;6:489-96.

13. Bardón-Otero E, Marti i Monros A, Vila Paz ML. Enfermería en la consulta de enfermedad renal crónica avanzada (ERCA). Nefrología 2008; (Supl. 3):S53-6.

14. Walker R, Abel S, Meyer A. Perceptions of key influences on effective pre-dialysis nursing care. Contemp Nurse. 2012;42(1):28-35.

15. García-Llana H, Remor E, Del Peso G, Selgas R. El papel de la depresión, la ansiedad, el estrés y la adhesión al tratamiento en la calidad de vida relacionada con la salud en pacientes en diálisis: revisión sistemática de la literatura. Nefrología 2014;34(5):637-57.

16. Malheiro Oliveira P, Arruda Soares D. Percepciones de las personas con insuficiencia renal crónica sobre la calidad de vida. Enfermería Global, 2012; $11(28): 257-75$

17. Sánchez-Cabezas AM, Gallego-Morillo N, Merino-Martínez RM, Crespo-Montero R. Calidad de vida de los pacientes en diálisis. Revisión sistemática. Enferm Nefrol. 2019;22(3):239-55.

18. Van Manen JG, Korevaar JC, Dekker W, Reuselaars MC, Boeschoten EW, Krediet RT, et al (The NECOSAD Study Group). Changes in employment status in End-Stage Renal Disease patients during their first year of dialysis. Peritoneal Dialysis Int. 2001;21:595-601. 
19. Nie $Y$, Witten $B$, Schatell $D$, Assari $S$, Ding $X$, Saran $R$, Bragg-Gresham JL. Changes in employment status prior to initiation of maintenance hemodialysis in the USA from 2006 to 2015. Clin Kidney J 2019;sfz077:1-8.

20. Erickson $K F$, Zhao B, Vivian $H$, Winkelmayer WC. Employment among Patients Starting Dialysis in the United States. Clin J Am Soc Nephrol. 2018; 13:265-73.

21. Bihong Huang, Bihong Lai, Ling $\mathrm{Xu}$, Ying Wang, Yanpei Cao, Ping Yan, Jing Chen. Low employment and low willingness of being reemployed in Chinese working-age maintained hemodialysis patients. Ren Fail. 2017; 39(1):607-12.

22. Dąbrowska-Bender M, Dykowska G, Żuk W, Milewska $M$, Staniszewska $A$. The impact on quality of life of dialysis patients with renal insufficiency. Patient Prefer Adherence 2018;12:577-83.
23. Huertas-Vieco MP, Pérez-García $R$, Albalate $M$, De Sequera $P$, Ortega M, Puerta M y cols. Factores psicosociales y adherencia al tratamiento farmacológico en pacientes en hemodiálisis crónica. Nefrología 2014;34(6):737-42.

24. Perales-Montilla CM, Duschek $S$, Reyes-del Paso $\mathrm{GA}$. Influencia de los factores emocionales sobre el informe de síntomas somáticos en pacientes en hemodiálisis crónica: relevancia de la ansiedad. Nefrología 2013;33(6):816-25.

25. Merino-Martínez RM, Gallego-Morillo N, Sánchez-Cabezas AM, Gómez-López VE, Crespo-Montero R. Relación entre la calidad de vida relacionada con la salud y la ansiedad/depresión en pacientes en hemodiálisis crónica. Enferm Nefrol. 2019;22(3):274-83.

Este artículo se distribuye bajo una Licencia Creative Commons Atribución-NoComercial 4.0 Internacional. https://creativecommons.org/licenses/by-nc/4.0/ 